\title{
Service Adaptable 3G Turbo Decoder for Indoor/Low Range Outdoor Environment
}

\author{
Costas CHAIKALIS, Nicholas S. SAMARAS \\ Department of Informatics \& Telecommunications, TEI of Larissa, Larissa, Greece \\ Email:kchaikalis@teilar.gr \\ Received July 28, 2009; revised September 6, 2009; accepted October 7, 2009
}

\begin{abstract}
For the well-known 3G mobile communications standard UMTS, four different service classes have been specified. Considering two turbo decoding algorithms, like SOVA and log-MAP, it would be desirable to use an efficient turbo decoder. In this paper this decoder is shown to adapt dynamically to different service scenarios, considering parameters like performance and complexity for indoor/low range outdoor operating environment. The scenarios show that for streaming service class real-time class applications the proposed decoding algorithm depends on data rate; for the majority of scenarios SOVA is proposed, whereas log-MAP is optimal for increased data rates and medium-sized frames. On the other hand, conversational service class real-time applications cannot be established. For the majority of non real-time applications (interactive and background service classes) either algorithm can be used, while log-MAP is proposed for medium data rates and frame lengths.
\end{abstract}

Keywords: Reconfigurable Systems, Turbo Decoder, UMTS, Flat Reyleigh Fading, Indoor/Low Range Outdoor Operating Environment

\section{Introduction and UMTS Data Flow}

Channel coding is a critical signal processing element in modern mobile communications systems. Turbo codes [1] represent a powerful channel coding technique. Universal Mobile Telecommunications System (UMTS) belongs to the third generation ( $3 \mathrm{G}$ ) of mobile communication systems. Turbo codes have been incorporated as a channel coding scheme in UMTS for data rates higher or equal to $28.8 \mathrm{kbps}$ [2]. They also provide high coding gains in flat fading channels with the use of outer block interleaving $[3,4]$. Soft-input/soft-output (SISO) decoder is part of a turbo decoder and two candidate algorithms to be used in a SISO decoder are soft output Viterbi algorithm (SOVA) and log maximum a-posteriori (logMAP) algorithm [2,5-7].

A reconfigurable turbo decoder can be derived according to the common operations of the two algorithms, optimal in terms of performance and latency $[8,9,10]$. We consider just SOVA and log-MAP and not other turbo decoding algorithms like max-log-MAP or MAP, because SOVA is better in terms of delay, while logMAP is better in terms of performance [3,5].

SOVA and log-MAP algorithms share common opera- tions which have been addressed in [8-10]. These common operations form a turbo decoder which can be reconfigured and choose the suitable turbo decoding algorithm for different applications (reconfigurable SOVA/ log-MAP turbo decoder). In [8] and [10] is also shown that in a reconfigurable SOVA/log-MAP turbo decoder scaling of the extrinsic information is possible with a common scaling factor, which is constant and independent of signal-to-noise ratio for additive white Gaussian noise (AWGN) channels. In [9] it is shown that in the case of a flat Rayleigh fading channel for a reconfigurable SOVA/log-MAP decoder a common scaling factor with value 0.7 is the optimal choice.

Nowadays, UMTS represents the dominant 3G system in the mobile communications market. According to UMTS specifications, a transport channel transfers data over radio interface from Medium Access Control sublayer of layer 2 to physical layer and is characterized by its transport format set, which consists of different transport formats. They must have the same type of channel coding and time transmission interval (TTI), while the transport block set or data frame size can vary. The transport block set determines the number of input bits to the channel encoder and can be transmitted every 
TTI, with possible values for TTI of 10, 20, 40 and 80 msec $[2,11]$. After channel coding, outer block interleaving is performed, and since the frame duration in UMTS is $10 \mathrm{msec}$, the number of columns of the outer block interleaver can be 1,2, 4 or 8 , depending on TTI value. Therefore, the TTI values and the number of columns of the outer block interleaver are interrelated. Furthermore, every transport channel is assigned a radio access bearer with a particular data rate, which provides the transfer of the service through the radio network. A mobile terminal may use several parallel transport channels simultaneously, each having its own characteristics (transport format set).

UMTS radio interface transfers multiple applications. Parameters like bit error rate (BER) performance and delay are assigned to these applications. Four different service traffic classes are defined: conversational, streaming, interactive and background. For real-time conversational and streaming classes BER has to be less than $10^{-3}$, while for non-real time interactive and background classes BER has to be less than $10^{-5}$. The maximum acceptable delay for conversational class is 80 msec, for streaming it is $250 \mathrm{msec}$, for interactive it is 1 $\mathrm{sec}$, while for background it is higher than $10 \mathrm{sec}[2,11]$.

\section{Simulation Parameters}

The discrete representation of flat Rayleigh fading channel is given by the following equation:

$$
y_{k}=\alpha_{k} \cdot x_{k}+n_{k}
$$

where $k$ is an integer symbol index, $x_{k}$ is a binary phase shift keying (BPSK) symbol amplitude $( \pm 1), n_{k}$ is a Gaussian random variable and $y_{k}$ is a noisy received symbol. The fading amplitude $a_{k}$ is a sample from a correlated Gaussian random process with zero mean and is generated using the Sum of Sines or Jakesmodel, which is described in [12]. This model is based on summing 9 sinusoids whose frequencies are chosen as samples of the Doppler spectrum. The properties of Jakes model are further analysed in [13].

For the simulation model a carrier frequency $f_{c}=2$

$\mathrm{GHz}$ is considered. It is also assumed that 1000000 bits are transmitted and grouped into frames whose length $k_{f}$ must be $\geq 40$ and $\leq 5114$, according to UMTS specifications $[2,14]$. For a particular transport channel, every TTI the data with the characteristics specified in a transport format of the transport channel ( $k_{f}$ bits $)$, is turbo encoded (constraint length $K=4$ and rate $\left.r_{c}=1 / 3\right)$ at the transmitter. Furthermore, each time instant it is assumed that the two recursive systematic convolutional encoders of the turbo encoder start encoding from all-zero state. After turbo coding and block interleaving using the UMTS parameters, the bits are BPSK modulated and transmitted through the mobile channel. At the receiver, outer block deinterleaving and turbo decoding is performed. The received values are not quantized which means that floating point arithmetic is used. The receiver is also assumed to have exact estimates of the fading amplitudes (perfect channel estimation without side information), while eight iterations are used in the turbo decoder.

Table 1 illustrates eight different UMTS dedicated transport channels with different transport format sets, which represent different implementation scenarios of the reconfigurable turbo decoder. The transport format set for each transport channel consists of different example transport formats and also of dynamic and semi-static parts. The semi-static part (turbo encoder parameters, TTI) is the same for all transport formats of the transport format set, while the dynamic part (frame size) differs [2, 11,15]. Moreover, as published simulation results have shown in $[3,4]$ for flat Rayleigh fading channels, data rate, outer block interleaving (thus TTI) and signal-tonoise ratio (SNR) greatly affect BER performance: for each scenario of Table 1 these three parameters differ considering also the examples presented in [15].

Table 1. Implementation scenarios.

\begin{tabular}{|c|c|c|c|c|c|c|c|}
\hline \multirow{4}{*}{$\begin{array}{l}\text { Transport } \\
\text { channel type }\end{array}$} & \multicolumn{4}{|c|}{ Transport format set } & \multirow{4}{*}{$\begin{array}{l}\text { Data rate } \\
R_{b}(k b p s)\end{array}$} & \multirow{4}{*}{$\begin{array}{l}\text { SNR } \\
(d B)\end{array}$} & \multirow{4}{*}{ Scenario } \\
\hline & \multirow{3}{*}{\begin{tabular}{|c|} 
Dynamic part \\
Transport block set or frame sizes (bits)
\end{tabular}} & \multicolumn{3}{|c|}{ Semi-static part } & & & \\
\hline & & \multicolumn{2}{|c|}{ Turbo encoder parameters } & \multirow{2}{*}{$\begin{array}{c}\text { TTI } \\
(\text { msec) }\end{array}$} & & & \\
\hline & & $\mathbf{K}$ & Code rate & & & & \\
\hline \multirow{8}{*}{$\begin{array}{l}\text { Dedicated } \\
\text { channel }\end{array}$} & 576,1152 & 4 & $1 / 3$ & 40 & 28.8 & 32 & 1 \\
\hline & $576,1152,1728,2304$ & 4 & $1 / 3$ & 40 & 57.6 & 30 & 2 \\
\hline & $336,672,1008,1344$ & 4 & $1 / 3$ & 20 & 64 & 30 & 3 \\
\hline & $336,672,1344,2688$ & 4 & $1 / 3$ & 20 & 128 & 30 & 4 \\
\hline & $336,672,1344,2688,3024$ & 4 & $1 / 3$ & 20 & 144 & 28 & 5 \\
\hline & $168,336,672,1344,2016,2688,3360,4032$ & 4 & $1 / 3$ & 20 & 384 & 28 & 6 \\
\hline & 2560 & 4 & $1 / 3$ & 40 & 64 & 30 & 7 \\
\hline & $336,1344,2688,4032,4704$ & 4 & $1 / 3$ & 40 & 2000 & 40 & 8 \\
\hline
\end{tabular}


Table 2. Quality of service and proposed decoding algorithm for scenarios 1, 2 and 3 of Table 1.

\begin{tabular}{|c|c|c|c|c|c|c|c|c|c|}
\hline & & $\begin{array}{c}\text { Frame size } \\
\text { (bits) }\end{array}$ & $\begin{array}{l}t_{d} \text { using SOVA } \\
\text { (msec) }\end{array}$ & $\underset{\text { (msec) }}{t_{d} \text { using log-MAP }}$ & $\begin{array}{l}\text { Max latency } \\
\quad(\text { msec) }\end{array}$ & $\begin{array}{l}\text { Log-MAP } \\
\text { BER }\end{array}$ & SOVA BER & $\begin{array}{l}\text { BER } \\
\text { range }\end{array}$ & $\begin{array}{c}\text { Proposed decoding } \\
\text { algorithm }\end{array}$ \\
\hline \multirow{6}{*}{$\begin{array}{c}\text { Scenario } \\
1\end{array}$} & \multirow{2}{*}{ Conv. class } & 576 & 240 & 528 & 80 & 0.000472 & 0.000523 & $<10^{-3}$ & \multirow{2}{*}{ Cannot be applied } \\
\hline & & 1152 & 400 & 976 & 80 & 0 & 0 & $<10^{-3}$ & \\
\hline & \multirow{2}{*}{$\begin{array}{c}\text { Strea } \\
\text { ming class }\end{array}$} & 576 & 240 & 528 & 250 & 0.000472 & 0.000523 & $<10^{-3}$ & \multirow{2}{*}{$\begin{array}{c}\text { SOVA } \\
\text { Cannot be applied }\end{array}$} \\
\hline & & 1152 & 400 & 976 & 250 & 0 & 0 & $<10^{-3}$ & \\
\hline & \multirow{2}{*}{$\begin{array}{c}\text { Non-real } \\
\text { time classes }\end{array}$} & 576 & 240 & 528 & Up to $1 \mathrm{sec}$ & 0.000472 & 0.000523 & $<10^{-5}$ & \multirow{2}{*}{$\begin{array}{l}\text { Cannot be applied } \\
\text { Log-MAP or SOVA }\end{array}$} \\
\hline & & 1152 & 400 & 976 & $\begin{array}{l}\text { interactive, }>10 \\
\text { sec background }\end{array}$ & 0 & 0 & $<10^{-5}$ & \\
\hline \multirow{12}{*}{$\begin{array}{c}\text { Scenario } \\
2\end{array}$} & \multirow{4}{*}{ Conv. class } & 576 & 160 & 304 & 80 & 0.001836 & 0.002096 & $<10^{-3}$ & \multirow{4}{*}{ Cannot be applied } \\
\hline & & 1152 & 240 & 528 & 80 & 0.000988 & 0.001036 & $<10^{-3}$ & \\
\hline & & 1728 & 320 & 752 & 80 & 0.000582 & 0.000634 & $<10^{-3}$ & \\
\hline & & 2304 & 400 & 976 & 80 & 0 & 0 & $<10^{-3}$ & \\
\hline & \multirow{4}{*}{$\begin{array}{c}\text { Strea } \\
\text { ming class }\end{array}$} & 576 & 160 & 304 & 250 & 0.001836 & 0.002096 & $<10^{-3}$ & \multirow{4}{*}{ Cannot be applied } \\
\hline & & 1152 & 240 & 528 & 250 & 0.000988 & 0.001036 & $<10^{-3}$ & \\
\hline & & 1728 & 320 & 752 & 250 & 0.000582 & 0.000634 & $<10^{-3}$ & \\
\hline & & 2304 & 400 & 976 & 250 & 0 & 0 & $<10^{-3}$ & \\
\hline & \multirow{4}{*}{$\begin{array}{c}\text { Non-real } \\
\text { time classes }\end{array}$} & 576 & 160 & 304 & \multirow{4}{*}{$\begin{array}{c}\text { Up to } 1 \mathrm{sec} \\
\text { interactive, }>10 \\
\text { sec background }\end{array}$} & 0.001836 & 0.002096 & $<10^{-5}$ & \multirow{3}{*}{ Cannot be applied } \\
\hline & & 1152 & 240 & 528 & & 0.000988 & 0.001036 & $<10^{-5}$ & \\
\hline & & 1728 & 320 & 752 & & 0.000582 & 0.000634 & $<10^{-5}$ & \\
\hline & & 2304 & 400 & 976 & & 0 & 0 & $<10^{-5}$ & Log-MAP or SOVA \\
\hline \multirow{12}{*}{$\begin{array}{c}\text { Scenario } \\
3\end{array}$} & \multirow{4}{*}{ Conv. class } & 336 & 82 & 157.6 & 80 & 0.003485 & 0.003888 & $<10^{-3}$ & \multirow{4}{*}{ Cannot be applied } \\
\hline & & 672 & 124 & 275.2 & 80 & 0.00146 & 0.00183 & $<10^{-3}$ & \\
\hline & & 1008 & 166 & 392.8 & 80 & 0.000779 & 0.000984 & $<10^{-3}$ & \\
\hline & & 1344 & 208 & 510.4 & 80 & 0.000519 & 0.000538 & $<10^{-3}$ & \\
\hline & \multirow{4}{*}{$\begin{array}{c}\text { Strea } \\
\text { ming class }\end{array}$} & 336 & 82 & 157.6 & 250 & 0.003485 & 0.003888 & $<10^{-3}$ & \multirow{2}{*}{ Cannot be applied } \\
\hline & & 672 & 124 & 275.2 & 250 & 0.00146 & 0.00183 & $<10^{-3}$ & \\
\hline & & 1008 & 166 & 392.8 & 250 & 0.000779 & 0.000984 & $<10^{-3}$ & SOVA \\
\hline & & 1344 & 208 & 510.4 & 250 & 0.000519 & 0.000538 & $<10^{-3}$ & SOVA \\
\hline & \multirow{4}{*}{$\begin{array}{c}\text { Non-real } \\
\text { time classes }\end{array}$} & 336 & 82 & 157.6 & & 0.003485 & 0.003888 & $<10^{-5}$ & \multirow{4}{*}{ Cannot be applied } \\
\hline & & 672 & 124 & 275.2 & $\begin{array}{l}\mathrm{Up} \text { to } 1 \mathrm{sec} \\
\end{array}$ & 0.00146 & 0.00183 & $<10^{-5}$ & \\
\hline & & 1008 & 166 & 392.8 & $\begin{array}{l}\text { interactive, }>10 \\
\text { sec background }\end{array}$ & 0.000779 & 0.000984 & $<10^{-5}$ & \\
\hline & & 1344 & 208 & 510.4 & & 0.000519 & 0.000538 & $<10^{-5}$ & \\
\hline
\end{tabular}

Table 3. Quality of service and proposed decoding algorithm for scenarios 4 and 5 of Table 1.

\begin{tabular}{|c|c|c|c|c|c|c|c|c|c|}
\hline & & $\begin{array}{l}\text { Frame size } \\
\text { (bits) }\end{array}$ & $\begin{array}{c}t_{d} \text { using SOVA } \\
(\mathrm{msec})\end{array}$ & $\underset{\text { (msec) }}{t_{d} \text { using log-MAP }}$ & $\begin{array}{l}\text { Max latency } \\
\quad(\text { msec) }\end{array}$ & $\begin{array}{c}\text { Log-MAP } \\
\text { BER }\end{array}$ & SOVA BER & $\begin{array}{l}\text { BER } \\
\text { range }\end{array}$ & $\begin{array}{l}\text { Proposed decoding } \\
\text { algorithm }\end{array}$ \\
\hline \multirow{11}{*}{$\begin{array}{c}\text { Scenario } \\
4\end{array}$} & \multirow{4}{*}{ Conv. class } & 336 & 61 & 98.8 & 80 & 0.003465 & 0.004047 & $<10^{-3}$ & \multirow{4}{*}{ Cannot be applied } \\
\hline & & 672 & 82 & 157.6 & 80 & 0.001584 & 0.001713 & $<10^{-3}$ & \\
\hline & & 1344 & 124 & 275.2 & 80 & 0.000796 & 0.000934 & $<10^{-3}$ & \\
\hline & & 2688 & 208 & 510.4 & 80 & 0 & 0 & $<10^{-3}$ & \\
\hline & \multirow{4}{*}{$\begin{array}{c}\text { Strea } \\
\text { ming class }\end{array}$} & 336 & 61 & 98.8 & 250 & 0.003465 & 0.004047 & $<10^{-3}$ & \multirow{4}{*}{ Cannot be applied } \\
\hline & & 672 & 82 & 157.6 & 250 & 0.001584 & 0.001713 & $<10^{-3}$ & \\
\hline & & 1344 & 124 & 275.2 & 250 & 0.000796 & 0.000934 & $<10^{-3}$ & \\
\hline & & 2688 & 208 & 510.4 & 250 & 0 & 0 & $<10^{-3}$ & \\
\hline & \multirow{3}{*}{$\begin{array}{c}\text { Non-real time } \\
\text { classes }\end{array}$} & 336 & 61 & 98.8 & \multirow{3}{*}{$\begin{array}{l}\text { Up to } 1 \mathrm{sec} \\
\text { interactive, } \\
>10 \mathrm{sec} \\
\text { background }\end{array}$} & 0.003465 & 0.004047 & $<10^{-5}$ & \multirow{3}{*}{ Cannot be applied } \\
\hline & & 672 & 82 & 157.6 & & 0.001584 & 0.001713 & $<10^{-5}$ & \\
\hline & & 1344 & 124 & 275.2 & & 0.000796 & 0.000934 & $<10^{-5}$ & \\
\hline
\end{tabular}




\begin{tabular}{|c|c|c|c|c|c|c|c|c|c|}
\hline & & 2688 & 208 & 510.4 & & 0 & 0 & $<10^{-5}$ & Log-MAP or SOVA \\
\hline \multirow{15}{*}{$\begin{array}{c}\text { Scenario } \\
5\end{array}$} & \multirow{5}{*}{ Conv. class } & 336 & 58.6 & 92.26 & 80 & 0.005771 & 0.006268 & $<10^{-3}$ & \multirow{5}{*}{ Cannot be applied } \\
\hline & & 672 & 77.3 & 144.5 & 80 & 0.003005 & 0.003287 & $<10^{-3}$ & \\
\hline & & 1344 & 114.6 & 249.06 & 80 & 0.000704 & 0.0010007 & $<10^{-3}$ & \\
\hline & & 2688 & 189.3 & 458.13 & 80 & 0 & $3.091 \mathrm{e}-05$ & $<10^{-3}$ & \\
\hline & & 3024 & 208 & 510.4 & 80 & 0 & 0 & $<10^{-3}$ & \\
\hline & \multirow{5}{*}{$\begin{array}{c}\text { Strea } \\
\text { ming class }\end{array}$} & 336 & 58.6 & 92.26 & 250 & 0.005771 & 0.006268 & $<10^{-3}$ & \multirow{2}{*}{ Cannot be applied } \\
\hline & & 672 & 77.3 & 144.5 & 250 & 0.003005 & 0.003287 & $<10^{-3}$ & \\
\hline & & 1344 & 114.6 & 249.06 & 250 & 0.000704 & 0.0010007 & $<10^{-3}$ & Log-MAP \\
\hline & & 2688 & 189.3 & 458.13 & 250 & 0 & $3.091 \mathrm{e}-05$ & $<10^{-3}$ & SOVA \\
\hline & & 3024 & 208 & 510.4 & 250 & 0 & 0 & $<10^{-3}$ & SOVA \\
\hline & \multirow{5}{*}{$\begin{array}{c}\text { Non-real time } \\
\text { classes }\end{array}$} & 336 & 58.6 & 92.26 & \multirow{5}{*}{$\begin{array}{l}\text { Up to } 1 \mathrm{sec} \\
\text { interactive, } \\
>10 \mathrm{sec} \\
\text { background }\end{array}$} & 0.005771 & 0.006268 & $<10^{-5}$ & \multirow{5}{*}{$\begin{array}{c}\text { Log-MAP } \\
\text { Log-MAP or SOVA }\end{array}$} \\
\hline & & 672 & 77.3 & 144.5 & & 0.003005 & 0.003287 & $<10^{-5}$ & \\
\hline & & 1344 & 114.6 & 249.06 & & 0.000704 & 0.0010007 & $<10^{-5}$ & \\
\hline & & 2688 & 189.3 & 458.13 & & 0 & $3.091 \mathrm{e}-05$ & $<10^{-5}$ & \\
\hline & & 3024 & 208 & 510.4 & & 0 & 0 & $<10^{-5}$ & \\
\hline
\end{tabular}

Table 4. Quality of service and proposed decoding algorithm for scenarios 6 and 7 of Table 1.

\begin{tabular}{|c|c|c|c|c|c|c|c|c|c|}
\hline & & $\begin{array}{l}\text { Frame size } \\
\text { (bits) }\end{array}$ & $\begin{array}{c}\mathbf{t}_{\mathrm{d}} \text { using SOVA } \\
(\mathrm{msec})\end{array}$ & $\begin{array}{c}t_{d} \text { using } \\
\text { log-MAP (msec) }\end{array}$ & $\begin{array}{l}\text { Max latency } \\
\quad(\text { msec })\end{array}$ & $\begin{array}{c}\text { Log-MAP } \\
\text { BER }\end{array}$ & SOVA BER & $\begin{array}{c}\text { BER } \\
\text { range }\end{array}$ & $\begin{array}{c}\text { Proposed decoding } \\
\text { algorithm }\end{array}$ \\
\hline \multirow{24}{*}{$\begin{array}{c}\text { Scenario } \\
6\end{array}$} & \multirow{8}{*}{ Conv. class } & 168 & 43.5 & 49.8 & 80 & 0.0073 & 0.008 & $<10^{-3}$ & \multirow{8}{*}{ Cannot be applied } \\
\hline & & 336 & 47 & 59.6 & 80 & 0.0065 & 0.007 & $<10^{-3}$ & \\
\hline & & 672 & 54 & 79.2 & 80 & 0.0051 & 0.0062 & $<10^{-3}$ & \\
\hline & & 1344 & 68 & 118.4 & 80 & 0.0022 & 0.0028 & $<10^{-3}$ & \\
\hline & & 2016 & 82 & 157.6 & 80 & 0.000654 & 0.001307 & $<10^{-3}$ & \\
\hline & & 2688 & 96 & 196.8 & 80 & 0 & 0 & $<10^{-3}$ & \\
\hline & & 3360 & 110 & 236 & 80 & 0 & 0 & $<10^{-3}$ & \\
\hline & & 4032 & 124 & 275.2 & 80 & 0 & 0 & $<10^{-3}$ & \\
\hline & \multirow{8}{*}{$\begin{array}{c}\text { Strea } \\
\text { ming class }\end{array}$} & 168 & 43.5 & 49.8 & 250 & 0.0073 & 0.008 & $<10^{-3}$ & \multirow{4}{*}{ Cannot be applied } \\
\hline & & 336 & 47 & 59.6 & 250 & 0.0065 & 0.007 & $<10^{-3}$ & \\
\hline & & 672 & 54 & 79.2 & 250 & 0.0051 & 0.0062 & $<10^{-3}$ & \\
\hline & & 1344 & 68 & 118.4 & 250 & 0.0022 & 0.0028 & $<10^{-3}$ & \\
\hline & & 2016 & 82 & 157.6 & 250 & 0.000654 & 0.001307 & $<10^{-3}$ & Log-MAP \\
\hline & & 2688 & 96 & 196.8 & 250 & 0 & 0 & $<10^{-3}$ & Log-MAP or SOVA \\
\hline & & 3360 & 110 & 236 & 250 & 0 & 0 & $<10^{-3}$ & Log-MAP or SOVA \\
\hline & & 4032 & 124 & 275.2 & 250 & 0 & 0 & $<10^{-3}$ & SOVA \\
\hline & \multirow{8}{*}{$\begin{array}{c}\text { Non-real time } \\
\text { classes }\end{array}$} & 168 & 43.5 & 49.8 & \multirow{8}{*}{$\begin{array}{l}\text { Up to } 1 \mathrm{sec} \\
\text { interactive, } \\
>10 \text { sec back- } \\
\text { ground }\end{array}$} & 0.0073 & 0.008 & $<10^{-5}$ & \multirow{8}{*}{$\begin{array}{l}\text { Log-MAP or SOVA } \\
\text { Log-MAP or SOVA } \\
\text { Log-MAP or SOVA }\end{array}$} \\
\hline & & 336 & 47 & 59.6 & & 0.0065 & 0.007 & $<10^{-5}$ & \\
\hline & & 672 & 54 & 79.2 & & 0.0051 & 0.0062 & $<10^{-5}$ & \\
\hline & & 1344 & 68 & 118.4 & & 0.0022 & 0.0028 & $<10^{-5}$ & \\
\hline & & 2016 & 82 & 157.6 & & 0.000654 & 0.001307 & $<10^{-5}$ & \\
\hline & & 2688 & 96 & 196.8 & & 0 & 0 & $<10^{-5}$ & \\
\hline & & 3360 & 110 & 236 & & 0 & 0 & $<10^{-5}$ & \\
\hline & & 4032 & 124 & 275.2 & & 0 & 0 & $<10^{-5}$ & \\
\hline \multirow[b]{3}{*}{$\begin{array}{c}\text { Scenario } \\
7\end{array}$} & Conv. class & 2560 & 400 & 976 & 80 & 0 & 0 & $<10^{-3}$ & Cannot be applied \\
\hline & $\begin{array}{c}\text { Strea } \\
\text { ming class }\end{array}$ & 2560 & 400 & 976 & 250 & 0 & 0 & $<10^{-3}$ & Cannot be applied \\
\hline & $\begin{array}{c}\text { Non-real time } \\
\text { classes }\end{array}$ & 2560 & 400 & 976 & $\begin{array}{l}\text { Up to } 1 \mathrm{sec} \\
\text { interactive, } \\
>10 \text { sec back- } \\
\text { ground }\end{array}$ & 0 & 0 & $<10^{-5}$ & Log-MAP or SOVA \\
\hline
\end{tabular}


Table 5. Quality of service and proposed decoding algorithm for scenario 8 of Table 1.

\begin{tabular}{|c|c|c|c|c|c|c|c|c|c|}
\hline & & $\begin{array}{c}\text { Frame size } \\
\text { (bits) }\end{array}$ & $\begin{array}{c}\mathbf{t}_{\mathrm{d}} \text { using SOVA } \\
\text { (msec) }\end{array}$ & $\underset{\text { (msec) }}{t_{d} \text { using log-MAP }}$ & $\begin{array}{l}\text { Max latency } \\
\quad(\text { msec) }\end{array}$ & $\begin{array}{c}\text { Log-MAP } \\
\text { BER }\end{array}$ & SOVA BER & $\begin{array}{c}\text { BER } \\
\text { range }\end{array}$ & $\begin{array}{c}\text { Proposed decoding } \\
\text { algorithm }\end{array}$ \\
\hline \multirow{15}{*}{$\begin{array}{c}\text { Scenario } \\
8\end{array}$} & \multirow{5}{*}{ Conv. class } & 336 & 81.34 & 83.76 & 80 & 0.001815 & 0.0019574 & $<10^{-3}$ & \multirow{5}{*}{ Cannot be applied } \\
\hline & & 1344 & 85.37 & 95.05 & 80 & 0.001414 & 0.0016548 & $<10^{-3}$ & \\
\hline & & 2688 & 90.75 & 110.1 & 80 & $9.97 \mathrm{e}-07$ & $9.97 \mathrm{e}-07$ & $<10^{-3}$ & \\
\hline & & 4032 & 96.12 & 125.1 & 80 & 0 & 0 & $<10^{-3}$ & \\
\hline & & 4704 & 98.81 & 132.68 & 80 & 0 & 0 & $<10^{-3}$ & \\
\hline & \multirow{5}{*}{$\begin{array}{c}\text { Strea } \\
\text { ming class }\end{array}$} & 336 & 81.34 & 83.76 & 250 & 0.001815 & 0.0019574 & $<10^{-3}$ & \multirow{2}{*}{ Cannot be applied } \\
\hline & & 1344 & 85.37 & 95.05 & 250 & 0.001414 & 0.0016548 & $<10^{-3}$ & \\
\hline & & 2688 & 90.75 & 110.1 & 250 & $9.97 \mathrm{e}-07$ & $9.97 \mathrm{e}-07$ & $<10^{-3}$ & Log-MAP or SOVA \\
\hline & & 4032 & 96.12 & 125.1 & 250 & 0 & 0 & $<10^{-3}$ & Log-MAP or SOVA \\
\hline & & 4704 & 98.81 & 132.68 & 250 & 0 & 0 & $<10^{-3}$ & Log-MAP or SOVA \\
\hline & \multirow{5}{*}{$\begin{array}{c}\text { Non-real } \\
\text { time classes }\end{array}$} & 336 & 81.34 & 83.76 & \multirow{5}{*}{$\begin{array}{l}\text { Up to } 1 \mathrm{sec} \\
\text { interactive, } \\
>10 \text { sec back- } \\
\text { ground }\end{array}$} & 0.001815 & 0.0019574 & $<10^{-5}$ & \multirow{2}{*}{ Cannot be applied } \\
\hline & & 1344 & 85.37 & 95.05 & & 0.001414 & 0.0016548 & $<10^{-5}$ & \\
\hline & & 2688 & 90.75 & 110.1 & & $9.97 \mathrm{e}-07$ & $9.97 \mathrm{e}-07$ & $<10^{-5}$ & Log-MAP or SOVA \\
\hline & & 4032 & 96.12 & 125.1 & & 0 & 0 & $<10^{-5}$ & Log-MAP or SOVA \\
\hline & & 4704 & 98.81 & 132.68 & & 0 & 0 & $<10^{-5}$ & Log-MAP or SOVA \\
\hline
\end{tabular}

According to [2] and [11], three different operating environments have been specified for UMTS:

- Rural outdoor operating environment with maximum supported mobile terminal speed $500 \mathrm{~km} / \mathrm{h}$ and maximum data rate of $144 \mathrm{kbps}$. Here, it has to be mentioned that a speed of $500 \mathrm{~km} / \mathrm{h}$ corresponds to high speed vehicles (e.g. trains). More typical value for this environment is $300 \mathrm{~km} / \mathrm{h}$.

- Urban or suburban outdoor operating environment with maximum supported mobile speed $120 \mathrm{~km} / \mathrm{h}$ and maximum data rate of $384 \mathrm{kbps}$.

- Indoor or low range outdoor operating environment with maximum supported mobile speed $10 \mathrm{~km} / \mathrm{h}$ and maximum data rate of $2 \mathrm{Mbps}$.

In [9] the approach is similar, but we considered the first two operating environments: a terminal speed of 300 $\mathrm{km} / \mathrm{h}$ for a rural outdoor environment and a terminal speed of $100 \mathrm{~km} / \mathrm{h}$ for an urban/suburban outdoor environment. In this paper we focus on the last operating environment and we choose a low terminal speed of 4 $\mathrm{km} / \mathrm{h}$. This means that the maximum data rate of $2 \mathrm{Mbps}$ can be considered. A terminal speed of $4 \mathrm{~km} / \mathrm{h}$ is a typical common value and it is important to be explored: represents walking human speed. In other words, each implementation scenario of the reconfigurable decoder of Table 1 is applied to indoor or low range outdoor operating environment. Moreover, similarly to $[8,9,10]$, for the calculation of total maximum delay per frame for SOVA and log-MAP we use the following equations assuming a pipeline turbo decoder architecture and a processor that runs at the same rate for both SOVA and log-MAP:
Total max delay using SOVA:

$$
t_{d}=2 \times T T I+\left(\frac{k_{f}}{R_{b}} \times N\right)
$$

Total max delay using log-MAP:

$$
t_{d}=2 \times T T I+\left(\frac{k_{f}}{R_{b}} \times N \times 2.8\right)
$$

where $t_{d}$ is the total delay, $k_{f}$ is the frame size, $R_{b}$ is the data rate of the radio bearer assigned to the transport channel and $N$ is the number of turbo decoder iterations. In these equations the higher complexity of log-MAP compared to SOVA (2.8 times) is also considered.

\section{Simulation Results}

The suitable decoding algorithm for each scenario is chosen according to performance and delay. Therefore, for each scenario of Table 1 all four service classes are applied to determine the quality of service profile parameters for different applications. Delay is calculated for each algorithm using Equations (2) and (3), while the simulated BER for each scenario is given in the following subsections together with a brief analysis of the results. Particularly, Table 2 shows quality of service for the different frame lengths of scenarios 1, 2, 3, while Tables 3 and 4 present quality of service for scenarios 4 , 5 and 6,7 , respectively. Finally, Table 5 presents quality of service for scenario 8 . 


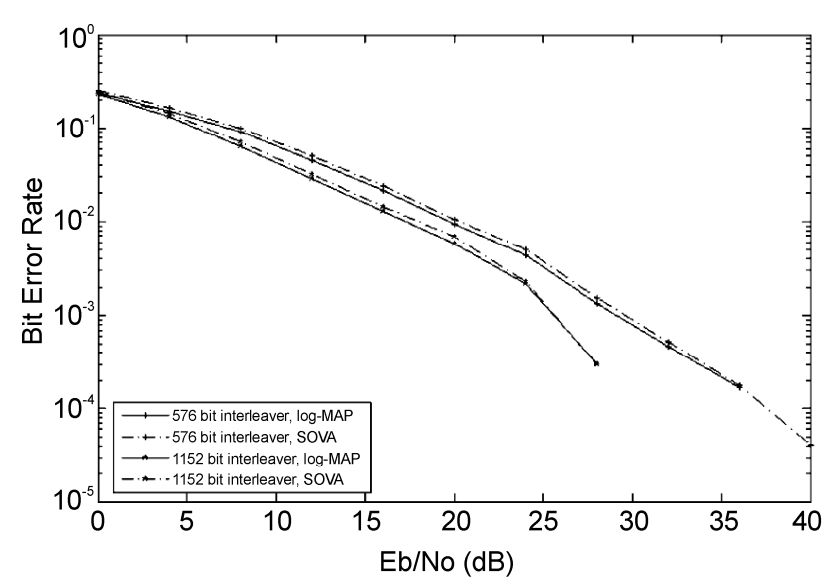

Figure 1. BER vs Eb/No for scenario 1.

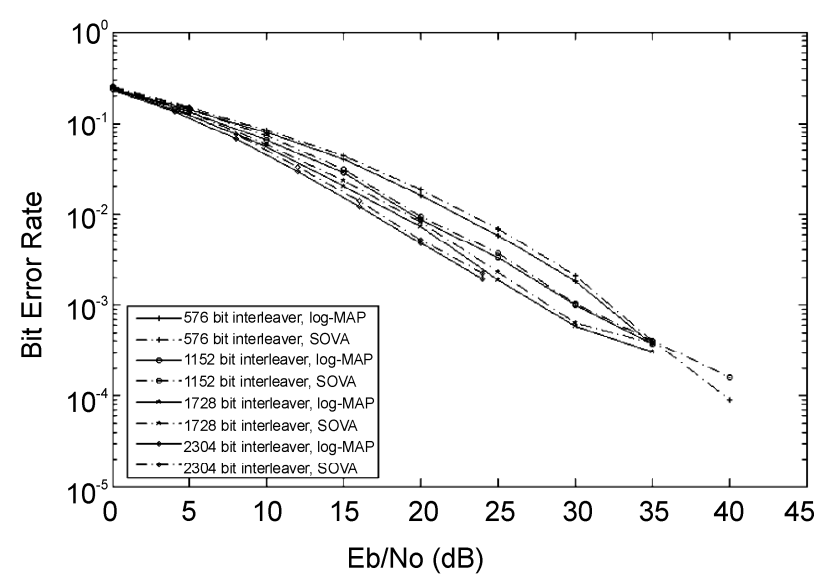

Figure 2. BER vs Eb/No for scenario 2.

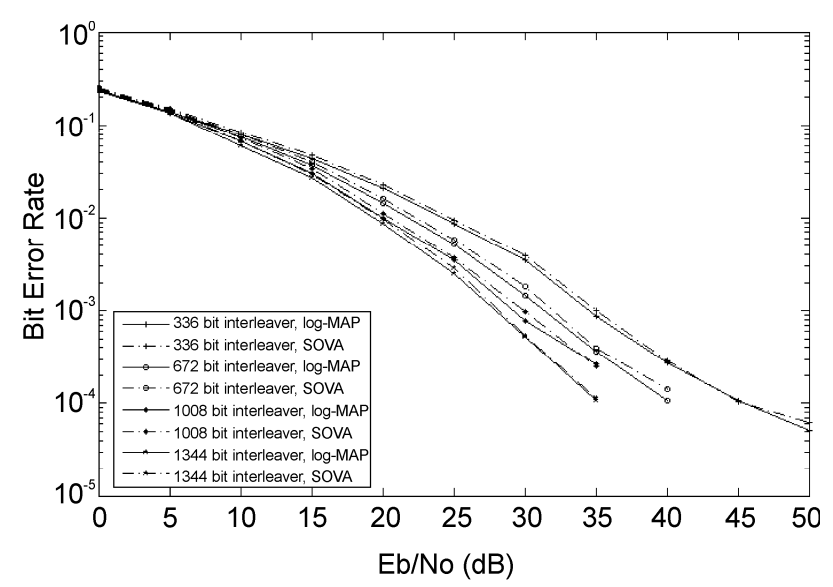

Figure 3. BER vs Eb/No for scenario 3.

\subsection{Scenario 1}

The simulated BER for this scenario is shown in Figure 1 assuming a symbol rate $R_{s}$ of $86.4 \mathrm{Kbaud}$, normalised fade rate $f_{d} T_{s}=0.000085$ with Doppler frequency $f_{d}=7.407 \mathrm{~Hz}$. Two frame lengths are considered in this scenario: 576 and 1152 bits, as Table 2 illustrates.

\subsubsection{Conversational Service Class}

At a SNR of $32 \mathrm{~dB}$, the conversational class cannot be considered for this scenario because even though the BER criterion is satisfied, latency is too high for all frame lengths for either SOVA or log-MAP.

\subsubsection{Streaming Service Class}

For this class only a frame length of 576 bits can be applied. In this case SOVA satisfies both requirements, while log-MAP exceeds the maximum acceptable delay limit. For a frame of 1152 bits delay for SOVA and $\log$-MAP is too high to achieve the limit for this class.

\subsubsection{Interactive/Background Service Classes}

For a frame length of 576 bits neither algorithm can be used because of the low BER criterion, while both requirements are achieved from both algorithms for a frame length of 1152 bits. Thus, a 576 bit frame service can not be applied, whereas in an 1152 bit frame service either SOVA or log-MAP can be used.

\subsection{Scenario 2}

The simulated BER results for this scenario are shown in Figure 2 assuming a symbol rate $R_{s}$ of $172.8 \mathrm{Kbaud}$, normalised fade rate $f_{d} T_{s}=0.000042$ and a SNR of 30 $\mathrm{dB}$.

\subsubsection{Conversational Service Class}

According to Table 2, for this class the four different frame lengths cannot be applied because of the tight delay limit ( $80 \mathrm{msec})$.

\subsubsection{Streaming Service Class}

Similarly, as illustrated in Table 2, the four frame lengths are not applicable. Particularly, for frame lengths of 576 and 1152 bits SOVA satisfies the delay criterion, but does not satisfy BER criterion. On the other hand, the use of log-MAP gives unacceptable delay. For frame lengths of 1728 and 2304 bits although BER is satisfied from both algorithms, maximum acceptable delay is exceeded.

\subsubsection{Interactive/Background Service Classes}

For these service classes it is well-known that BER must be low and latency limits are not very strict. Thus, the first three frame lengths cannot be applied due to not acceptable BER. For a frame length of 2304 bits the two criteria are achieved by both decoding algorithms: either SOVA or log-MAP can be used. 


\subsection{Scenario 3}

Figure 3 presents the simulation results for this scenario using the following parameters: $R_{s}=192$ Kbaud, $f_{d} T_{s}=0.000038$ and a SNR of $30 \mathrm{~dB}$.

\subsubsection{Conversational Service Class}

According to the analysis of Table 2, the four frame lengths give too high delay. Thus, their application is not possible for SOVA or log-MAP.

\subsubsection{Streaming Service Class}

The analysis of Table 2 clearly shows that for all frame lengths SOVA satisfies the delay limit of $250 \mathrm{msec}$ at 30 $\mathrm{dB}$. On the other hand the BER limit is not achieved for the small frames of 336 and 672 bits. Thus, SOVA can be used for frames of 1008 and 1344 bits. For log-MAP and frames of $672,1008,1344$ bits the delay limit cannot be achieved. For a small frame of 336 bits the delay limit is achieved, but the BER limit is not achieved.

\subsubsection{Interactive/Background Service Classes}

For these non-real time service classes and for all four frames the achieved BER is lower than the acceptable limit. Therefore, although the delay limit is achieved the four frames can not be applied.

\subsection{Scenario 4}

Figure 4 presents the simulated BER for this scenario using the following parameters: $R_{s}=384$ Kbaud, $f_{d} T_{s}=0.000019$ with $f_{d}=185.1 \mathrm{~Hz}$ and a SNR of 30 $\mathrm{dB}$.

\subsubsection{Conversational Service Class}

Again, for this class the four frames cannot be applied

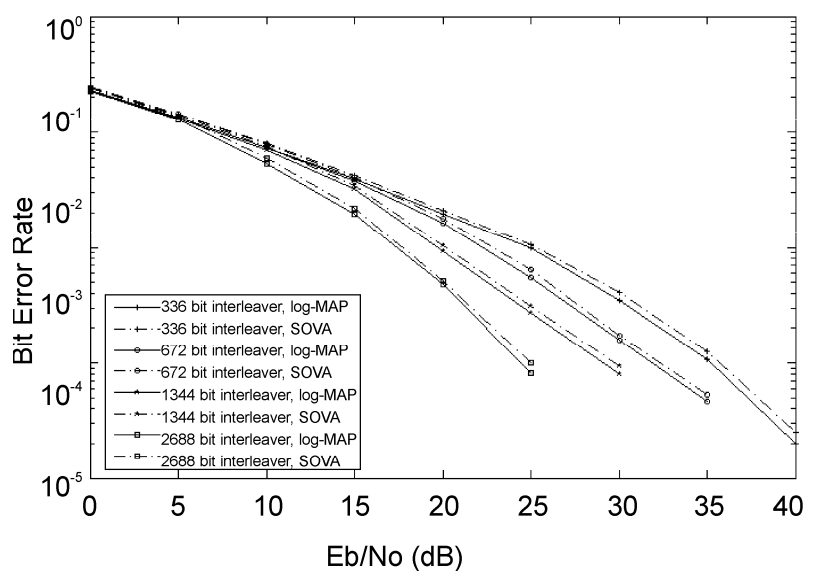

Figure 4. BER vs Eb/No for scenario 4. because of high delay. For a frame of 336 bits although delay is acceptable for SOVA, BER criterion is not satisfied. According to Table 3 it is obvious that this service scenario is not possible to be implemented.

\subsubsection{Streaming Service Class}

The analysis of Table 3 clearly shows that for all frame lengths SOVA satisfies the delay limit of $250 \mathrm{msec}$ at 30 $\mathrm{dB}$. On the other hand the BER limit is not achieved for the small frames of 336 and 672 bits. Thus, SOVA is the proposed turbo decoding algorithm for frames of 1344 and 2688 bits. For log-MAP and frames of 1344, 2688 bits the delay limit cannot be achieved. For small frames of 336 and 672 bits the delay limit is achieved, but the BER limit is not achieved.

\subsubsection{Interactive/Background Service Classes}

According to Table 3, for these classes and for the first three frames the achieved BER is lower than the acceptable limit. Therefore, although the delay limit is achieved these frames can not be applied. On the other hand, for a frame of 2688 bits the two parameters (BER, delay) are satisfied by both algorithms.

\subsection{Scenario 5}

For Figure 5 the following parameters are assumed: $R_{s}=432$ Kbaud, $f_{d} T_{s}=0.000017$ and a SNR of 28 dB. Figure 5 shows BER performance for the five different frame lengths specified in Table 1 for this scenario.

\subsubsection{Conversational Service Class}

For this class (Table 3) for all five frames the delay criterion is too low to be achieved from both algorithms. There is an exception for the small frames of 336 and 672 bits, where the delay criterion is achieved for SOVA but BER criterion is not. It is obvious that the constraints

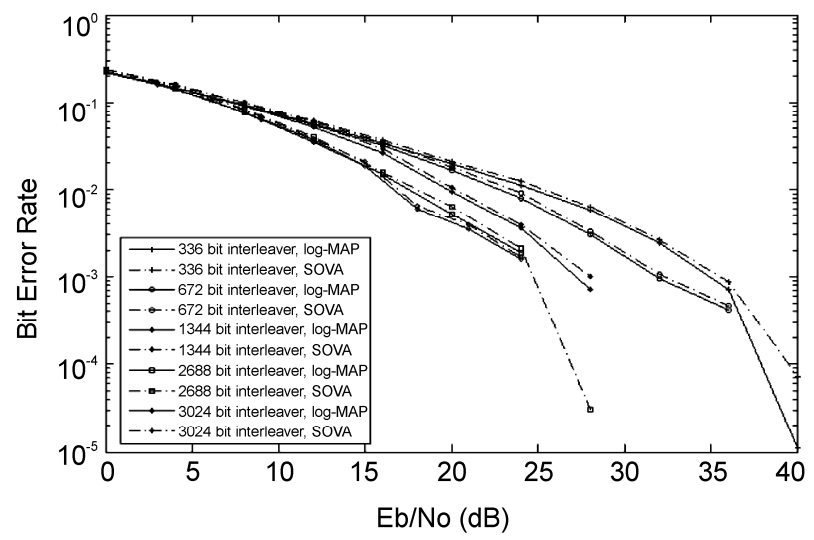

Figure 5. BER vs Eb/No for scenario 5. 
of the two parameters cannot be achieved by both algorithms.

\subsubsection{Streaming Service Class}

The analysis of Table 3 identifies three cases:

- Small frames of 336 and 672 bits. Here, the delay limit is achieved, but the BER limit is not for SOVA and log-MAP. This means that these frames cannot be implemented.

- Medium frame of 1152 bits. Here, the delay limit is achieved by both algorithms. Log-MAP is the proposed choice because it can achieve the BER limit as well. SOVA cannot achieve the BER limit. Thus, log-MAP represents the proposed algorithm.

- Large frames of 2688 and 3024 bits. Here, SOVA is the algorithm that can be implemented. The reason is the following: BER limit is achievable by both algorithms, whereas delay limit is achieved only by SOVA.

\subsubsection{Interactive/Background Service Classes}

According to Table 3, the delay limit is achieved by both SOVA and log-MAP for all frames. Furthermore, for the first three frames the BER limit is not achieved, but for 2688 bits frame it is achieved only by log-MAP. In this case log-MAP is proposed. For a frame of 3024 bits the limits of the two parameters are achieved by both algorithms.

\subsection{Scenario 6}

Figure 6 illustrates the simulated BER of the different frame lengths for this scenario using the following parameters: $R_{s}=1152$ Kbaud, $f_{d} T_{s}=0.0000064$ and a SNR of $28 \mathrm{~dB}$.

\subsubsection{Conversational Service Class}

For this class (Table 4) for the first three frames although delay criterion is satisfied, BER criterion is not satisfied.

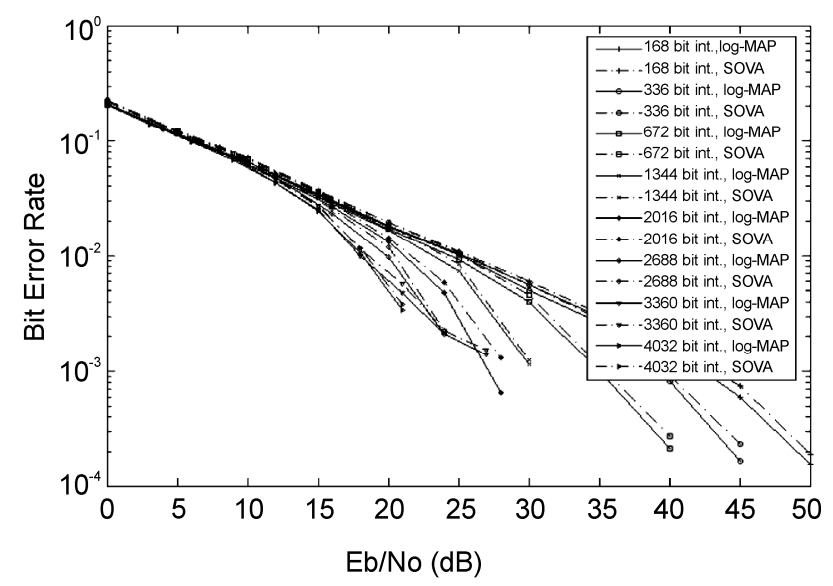

Figure 6. BER vs Eb/No for scenario 6.
For the next four frames either BER, or delay limits are not achieved for SOVA and log-MAP. Thus, this service class is not possible to be implemented for all frames.

\subsubsection{Streaming Service Class}

The analysis of Table 4 identifies four cases:

- $\quad$ Frames of 168, 336, 672 and 1344 bits. Here, the delay limit is achieved, but the BER limit is not achieved for SOVA and log-MAP. This means that these frames cannot be implemented.

- $\quad$ Frame of 2016 bits. Here, the delay limit is achieved by both algorithms. Log-MAP is the proposed choice because it can achieve the BER limit as well, while SOVA cannot achieve the BER limit. Thus, logMAP represents the proposed algorithm.

- $\quad$ Frames of 2688 and 3360 bits. Here, SOVA and log-MAP achieve both limits. Therefore, both algorithms can be used.

- $\quad$ Frame of 4032 bits. Here, SOVA is the algorithm that can be implemented. The reason is the following: BER limit is achievable by both algorithms, whereas log-MAP gives unacceptable delay.

\subsubsection{Interactive/Background Service Classes}

According to Table 4, the delay limit is achieved by both SOVA and log-MAP for all frames. Furthermore, for the first five frames the BER limit is not achieved. Thus, they cannot be implemented. For frames of 2688, 3360 and 4032 bits the limits of the two parameters are achieved by both algorithms.

\subsection{Scenario 7}

In Figure 7 BER performance for the different frame lengths for this scenario can be seen using the following parameters: $R_{s}=192$ Kbaud, $f_{d} T_{s}=0.000038$ and a SNR of $30 \mathrm{~dB}$.

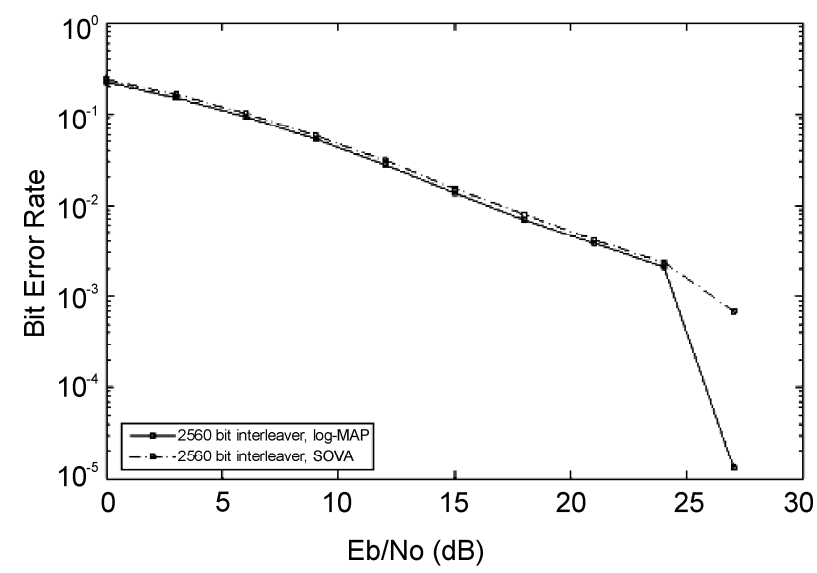

Figure 7. BER vs Eb/No for scenario 7. 


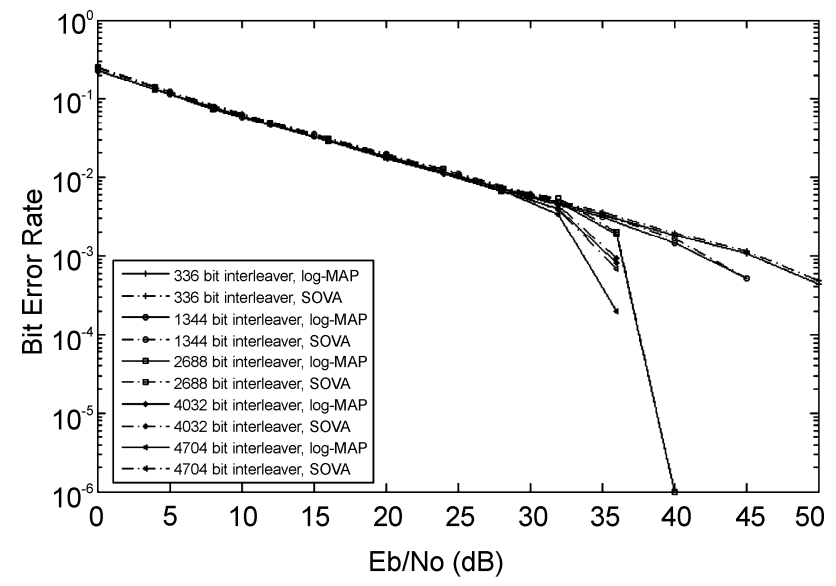

Figure 8. BER vs Eb/No for scenario 8.

\subsubsection{Conversational/Streaming Service Classes}

The analysis of Table 4 clearly shows that the frame of 2560 bits gives unacceptable delay for both real time classes and both decoding algorithms. Therefore, they cannot be implemented.

\subsubsection{Interactive/Background Service Classes}

For non-real time classes both limits are achieved by both algorithms, which mean that they are both suitable for this application.

\subsection{Scenario 8}

Figure 8 presents BER performance of the different frame lengths for this scenario using the following parameters: $R_{s}=6000$ Kbaud, $f_{d} T_{s}=0.0000012$ and a SNR of $40 \mathrm{~dB}$.

\subsubsection{Conversational Service Class}

For all five frames the calculated delay, according to Table 5, is too high. Thus, this scenario cannot be implemented for this service class.

\subsubsection{Streaming Service Class}

Here, delay criterion is achieved by both algorithms and for all frames. Furthermore, for frames of 336 and 1344 bits the BER limit is not achievable by the two algorithms. This means that these two frames cannot be implemented. On the other hand, for frames of 2688, 4032 and 4704 bits the two criteria are satisfied by both algorithms: they are equally suitable.

\subsubsection{Interactive/Background Service Classes}

From Table 5 it can be seen that the analysis is similar to the previous section: the first two frames cannot be established, whereas for the last three frames either SOVA or log-MAP can be used.

\section{Conclusions}

In this paper we have presented possible reconfiguration scenarios applied to an important receiver technique, namely, channel decoding. It has been shown that reconfigurability is a desirable feature towards the implementation of energy efficient receivers without performance sacrifices.

For a UMTS turbo decoder SOVA and log-MAP correspond to the main decoding algorithms. Considering performance and complexity or delay, SOVA is the best choice in terms of complexity, while log-MAP is the best choice in terms of performance. The similarities in the data-flow of the two algorithms support the idea of a reconfigurable SOVA/log-MAP turbo decoder $[8,9,10]$. Moreover, according to [3] at low terminal speeds BER is worse than at higher terminal speeds. For UMTS some applications require the lowest possible delay, while for others the lowest possible performance is sufficient. Having in mind the results of [9] it is observed that at rural and urban/suburban outdoor operating environments more frames can be established compared to indoor/low range outdoor environment. Thus, for indoor/ low range outdoor environment there are many applications which cannot be established.

Our results for indoor/low range outdoor environment show that for all implementation scenarios real time conversational class cannot be established. The reason is the low terminal speed which gives high BER. Comparing with urban/suburban environment in [9], this class can be applied to medium sized frames and high data rates, whereas in rural outdoor operating environment this class can be applied to small frames and low or medium data rates.

For real time streaming class the proposed algorithm choice depends on data rate. For low data rates all frames cannot be applied, except for small frames where SOVA is optimal. For medium data rates (64 kbps, $128 \mathrm{kbps}$ ) small frames cannot be applied, while for medium-sized frames SOVA is proposed. For $144 \mathrm{kbps}$ again SOVA is proposed for larger frames, while for medium-sized frames log-MAP is optimal. For high data rates (384 kbps) small frames cannot be considered, for medium frames log-MAP is proposed, while for large frames SOVA is proposed. For the other frame lengths either algorithm is proposed. For very high data rates ( $2 \mathrm{Mbps})$ small frames cannot be established: for the other frames either SOVA or log-MAP can be used. On the other hand, in [9] for streaming class applications urban/suburban and rural outdoor operating environments SOVA is optimal for the scenarios that can be established. It is remarkable that, similarly to [9], as data rate increases more and larger frames can be applied.

For non-real time applications performance is the priority and delay requirements are looser. We observe that 
for all scenarios small frames cannot be applied due to tight BER. For larger frames both algorithms are equally suitable. Furthermore, for medium data rates and medium frames log-MAP is the proposed algorithm choice. For urban/suburban outdoor environment the conclusions are similar in [9], whereas for rural outdoor environment log-MAP is optimum for the small frames and the two algorithms are equally suitable for larger frames.

\section{References}

[1] C. Berrou and A. Glavieux, "Near optimum error correcting coding and decoding: Turbo codes," IEEE Trans. on Communications, Vol. 44, No. 10, pp. 1261-1271, 1996.

[2] H. Holma and A. Toskala, "WCDMA for UMTS: Radio access for third generation mobile communications," J. Wiley, 2000.

[3] J. Woodard and L. Hanzo, "Comparative study of turbo decoding techniques: An overview," IEEE Transactions on Vehicular Technology, Vol. 49, No. 6, pp. 2208-2233, 2000.

[4] E. Hall and S. Wilson, "Design and analysis of turbo codes on Rayleigh fading channels," IEEE Journal on Selected Areas in Communications, Vol. 16, No. 2, pp. 160-174, 1998.

[5] P. Robertson, E. Villebrun, and P. Hoeher, "A comparison of optimal and sub-optimal MAP decoding algorithms operating in the log domain," IEEE ICC'95, Seat- tle, USA, pp. 1009-1013, 1995.

[6] J. Hagenauer and P. Hoher, "A Viterbi algorithm with soft outputs and its applications," IEEE GLOBECOM'89, Dallas, USA, pp. 1680-1686, 1989.

[7] S. Pietrobon, "Implementation and performance of a turbo/MAP decoder," International Journal of Satellite Communications, Vol. 16, No. 1, pp. 23-46, 1998.

[8] C. Chaikalis, "Reconfigurable structures for turbo codes in $3 \mathrm{G}$ mobile radio transceivers," $\mathrm{PhD}$ thesis, School of Engineering Design and Technology, University of Bradford, UK, 2003.

[9] C. Chaikalis, "Implementation of a reconfigurable turbo decoder in 3GPP for flat Rayleigh fading," Elsevier Digital Signal Processing Journal, 2008.

[10] C. Chaikalis and J. M. Noras, "Reconfigurable turbo decoding for 3G applications," Elsevier Signal Processing Journal, Vol. 84, No. 10, pp. 1957-1972, 2004.

[11] 3GPP TS 25.201 V3.3.0. "Physical layer-General description," Release 1999, 2002.

[12] W. C. Jakes, "Microwave mobile communications," J. Wiley \& Sons, New York, 1974.

[13] M. Patzold, U. Killat, F. Laue, and Y. Li, "On the statistical properties of deterministic simulation models for mobile fading channels," IEEE Transactions on Vehicular Technology, Vol. 47, No. 1, pp. 254-269, 1998.

[14] 3GPP TS 25.212 V3.9.0. "Multiplexing and channel coding (FDD)," Release 1999, 2002.

[15] 3GPP TR 25.944 V3.5.0. "Channel coding and multiplexing examples," Release 1999, 2001. 